\title{
COMPARAÇÃO DOS EXAMES COMPLEMENTARES NO DIAGNÓSTICO DAS LESÕES DO MANGUITO ROTADOR
}

\author{
COMPARISON OF COMPLEMENTARY EXAMS IN THE \\ DIAGNOSIS OF ROTATOR CUFF INJURIES
}

Gabriel El-Kouba, Thomas Andreas Huber, José Renato Wilke Freitas, Valdir Steglich, Henrique Ayzemberg, Adriano M. Santos

\section{RESUMO}

Objetivo: A proposta do trabalho é avaliar a acurácia da radiografia simples, ultrassonografia e ressonância nuclear magnética no diagnóstico das lesões do manguito rotador, comparando-os com os achados em cirurgias abertas ou artroscópicas. Métodos: Foram avaliados protocolos do Serviço de Cirurgia do Ombro e Cotovelo dos pacientes submetidos ao tratamento cirúrgico por lesão de manguito rotador diagnosticados por radiografia, ultrassom e/ou ressonância no período de 2002-2007. Com base nos dados coletados, analisamos a sensibilidade, especificidade, valor preditivo positivo, valor preditivo negativo e acurácia destes exames complementares, comparados aos achados durante os procedimentos cirúrgicos. Resultados: Foram incluídos no estudo 147 pacientes, com média de idade de 46,09 anos. Todos os pacientes estudados haviam realizado um exame de radiografia, 101 apresentavam ultrassonografia e 72 pacientes realizaram ressonância magnética. Encontramos sensibilidade de $13,8 \%$, especificidade de $2,6 \%$ e acurácia de $30 \%$ com a radiografia; sensibilidade de 57,6\%, especificidade de 29,6 e acurácia de 51,4\% com o ultrassom; e sensibilidade de 86,6\%, especificidade de $22,2 \%$ e acurácia de $63,3 \%$ com a ressonância. Conclusão: A radiografia se mostrou um exame específico quando apresentou sinal do espelho. A ressonância e a ultrassonografia se mostraram métodos confiáveis apresentando elevada acurácia no diagnóstico das lesões do manguito rotador.

Descritores - Bainha Rotadora; Ultrassonografia; Ressonância Magnética; Diagnóstico

\section{ABSTRACT}

Objective: This paper proposes to evaluate the accuracy of $x$-rays, ultrasounds, and nuclear magnetic resonance in the diagnosis of rotator cuff injuries, comparing their findings with open surgery or arthroscopic findings. Methods: Shoulder and Elbow Surgery Department protocols for patients undergoing surgical treatment for rotator cuff injury diagnosed by $x$-ray, ultrasound and/or resonance in our clinic, that were between 2002 and 2007. Based on the data collected, we analyzed the sensitivity, specificity, positive predictive value, negative predictive value, and accuracy of these complementary exams, compared to the findings from surgical procedures. Results: The study included 147 patients with a mean age of 46.09 years. Every patient had completed an x-ray exam, 101 had had an ultrasound and 72 patients underwent magnetic resonance. We found a sensitivity of $13.8 \%$, specificity of $2.6 \%$, and accuracy of $30 \%$ with the $x$-rays; a sensitivity of $57.6 \%$, specificity of $29.6 \%$, and accuracy of $51.4 \%$ with the ultrasound; and a sensitivity of $86.6 \%$, specificity of $22.2 \%$, and accuracy of $63.3 \%$ with magnetic resonance. Conclusions: X-rays showed specificitywhen the mirror sign was present. Magnetic resonance and ultrasound demonstrated themselves to be reliable methods with high accuracy for rotator cuff injury diagnosis.

Keywords - Rotator Cuff; Ultrasonography; Resonance Magnetic Image; Diagnosis

\section{INTRODUÇÃO}

As lesões do manguito rotador constituem uma causa frequente de dor no ombro em pessoas de todas as idades. Essa condição representa um espectro de doenças, que varia desde uma tendinite aguda reversível até uma lesão maciça envolvendo todos os seus componentes ${ }^{(1)}$.

O diagnóstico dessa doença é obtido pela história clínica e exame físico, incluindo suas manobras semio-

Preceptores do Serviço de Residência Médica do Instituto de Ortopedia e Traumatologia (IOT) - Joinville, SC.

Trabalho realizado no Instituto de Ortopedia e Traumatologia (IOT) - Joinville, SC

Correspondência: Rua Blumenau, 1.316 Bairro América - 89204-251 - Joinville, SC, Brasil. E-mail:gabrielkouba@hotmail.com 
lógicas específicas e mediante a infiltração de anestésicos locais no espaço subacromial em casos de dúvida diagnóstica (testes específicos) $)^{(2)}$.

Os exames de radiografia auxiliam na definição da causa do impacto, avaliando fatores anatômicos como a forma e inclinação do acrômio, presença de esporões acromiais, os acromiale e osteoartrose da articulação acromioclavicular. Evidenciam alterações secundárias da síndrome do impacto, como cistos subcondrais, esclerose e irregularidades da grande tuberosidade do úmero. As radiografias servem ainda para identificar calcificações tendíneas e peritendíneas ${ }^{(2)}$.

Os principais métodos não invasivos utilizados no diagnóstico das lesões do manguito rotador são a ultrassonografia e a ressonância nuclear magnética ${ }^{(3,4)}$. A ultrassonografia apresenta um custo menor, mas os resultados quanto à sensibilidade e especificidade variam por se tratar de um exame "operador-dependente"(4-6). A ressonância demonstra o contraste entre tecidos ósseos e partes moles, apresentando lesões degenerativas e rupturas tendíneas além de outras anormalidades estruturais eventualmente presentes ${ }^{(7,8)}$.

Existem poucos relatos na literatura que comparam a precisão da ultrassonografia e ressonância magnética no diagnóstico das lesões do manguito rotador e na quantificação do tamanho da lesão. Tanto a detecção quanto a mensuração da lesão podem ajudar na indicação da modalidade terapêutica cirúrgica aberta ou artroscópica, assim como dar indícios do prognóstico pós-reparo dessas lesões ${ }^{(9-11)}$.

O objetivo deste trabalho é avaliar a acurácia da radiografia simples, ultrassonografia e ressonância nuclear magnética no diagnóstico das lesões do manguito rotador do ombro, comparando-os com os achados em cirurgias abertas ou artroscópicas como padrão.

\section{MÉTODOS}

Foram avaliados protocolos do Serviço de Cirurgia do Ombro e Cotovelo (Anexo 1) do Serviço de Residência Médica, no período de 2002-2007, através da análise de todos os pacientes submetidos ao tratamento cirúrgico por doenças do ombro. Todos os pacientes foram operados por dois cirurgiões ortopédicos instrutores do Serviço de Residência Médica.

Neste estudo foram incluídos pacientes adultos, de ambos os sexos, com diagnóstico clínico e de imagem de lesão do manguito rotador, tratados cirurgicamente após falhas nas tentativas de tratamento conservador conforme protocolo do serviço.
A média de idade dos pacientes avaliados foi de 46,09 anos, tendo 20 anos o paciente mais novo e 69 anos o mais velho. Houve uma prevalência do sexo masculino, com 83 pacientes $(56,5 \%)$, contra 64 mulheres $(43,5 \%)$.

Todos os pacientes realizaram avaliação radiográfica pelas incidências anteroposterior verdadeiro, perfil escapular e axilar e posteriormente foram submetidos à ultrassonografia, ressonância magnética, ou ambas.

Todos os exames de ultrassonografia e ressonância foram realizados em uma das duas clínicas especializadas situadas na cidade da instituição realizadora do trabalho, sendo analisadas e laudadas por médicos radiologistas especialistas na área musculoesquelética.

As avaliações das radiografias foram realizadas pelos próprios cirurgiões. As impressões diagnósticas da análise da radiografia foram divididas da seguinte maneira: 1) sem alterações; 2) esclerose e cistos subcondrais na região da grande tuberosidade; e 3) "sinal do espelho", com formação osteofitária na região anteroinferior do acrômio.

No estudo dos exames de ultrassonografia foram adotados os seguintes critérios para diagnóstico de ruptura do manguito rotador: não visualização de um ou mais tendões do manguito; não visualização focal em um dos tendões e defeito ou descontinuidade bem definida no tendão que atingisse as duas faces do tendão (articular e bursal). Para caracterização da lesão vista à ultrassonografia, consideramos três possibilidades: 1) normal (sem lesão); 2) lesão parcial; e 3) lesão total de tendão/tendões do manguito rotador.

$\mathrm{Na}$ avaliação da ressonância nuclear magnética os critérios adotados análise do manguito rotador foram os mesmos descritos no protocolo de Slatkin. Este determina quatro estágios da lesão do manguito rotador na ressonância magnética: 0 - tendão de sinal e morfologia normal; 1 - aumento de intensidade de sinal sem irregularidade ou descontinuidade; 2 - aumento de sinal com irregularidade e afilamento do tendão; e 3 perda completa de solução do tendão ${ }^{(12)}$. Com base nos laudos descritos através da utilização deste protocolo, distribuímos os pacientes em grupos conforme os resultados da ressonância magnética: 1) normal (sem lesão); 2) lesão parcial; e 3) lesão total do tendão.

$\mathrm{O}$ tratamento cirúrgico por via artroscópica ou mista foi realizado em decúbito lateral. Neste caso, a avaliação da lesão foi realizada por via artroscópica; porém, o reparo da lesão foi realizado por via aberta. Durante a cirurgia foi feita uma avaliação do manguito rotador, objetivando 
identificar rupturas e alterações degenerativas.

No transoperatório, os pacientes foram classificados como: 1) sem rupturas ou lesões; 2) rupturas parciais, sendo realizado desbridamento das lesões; e 3) lesões totais, com o reparo das mesmas quando possível.

Com base nos dados coletados, analisamos a sensibilidade, especificidade, valor preditivo positivo, valor preditivo negativo e acurácia da radiografia simples, ultrassonografia e ressonância magnética para o diagnóstico de qualquer lesão de manguito rotador. Analisamos também a capacidade destes três exames complementares, através dos mesmos indicadores acima citados, no diagnóstico específico das lesões parciais e totais do manguito rotador, assim como para a ausência de lesão dos tendões.

\section{RESULTADOS}

Foram analisados 454 protocolos de pacientes submetidos à cirurgia do ombro no período de 2002 a 2007. Destes, 187 pacientes tiveram a lesão do manguito rotador como diagnóstico com indicação de tratamento cirúrgico. Foram excluídos do estudo 40 pacientes por dados incompletos e foram incluídos no estudo 147 pacientes.

Todos os pacientes estudados haviam realizado um exame de radiografia, e tivemos 13 pacientes com imagem de "sinal do espelho" (8,85\%), exame normal em três $(2,05 \%)$ e $131(89,1 \%)$ com alguma alteração radiográfica, como esclerose de grande tuberosidade ou cistos subcondrais (Figura 1).

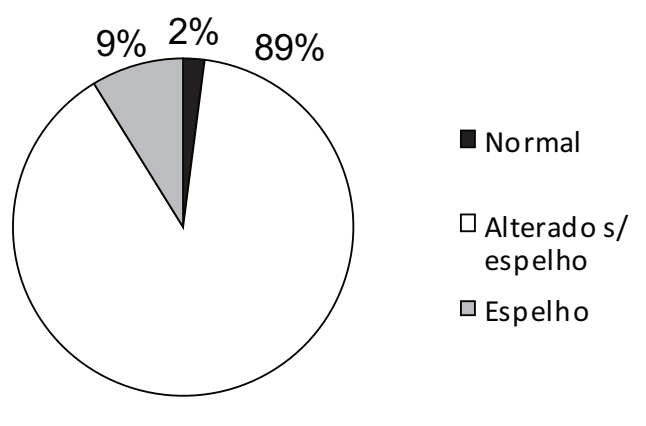

Figura 1 - Distribuição dos pacientes segundo a presença de alteração na radiografia (valores percentuais aproximados).

De todos os protocolos analisados, 101 apresentavam dados referentes à ultrassonografia realizada. Destes, havia 22 pacientes $(21,8 \%)$ com exame normal, 43 $(42,6 \%)$ com lesão parcial do manguito rotador e 36 $(35,6 \%)$ com diagnóstico de lesão completa/total do manguito (Figura 2).

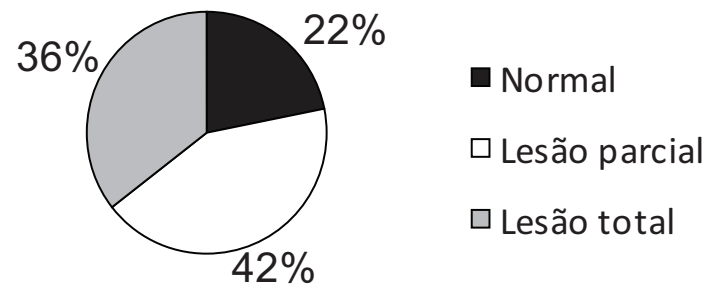

Figura 2 - Distribuição dos pacientes segundo a presença de alterações na ultrassonografia (valores percentuais aproximados).

Apenas 72 pacientes realizaram ressonância magnética. Destes, tivemos 34 (47,2\%) com conclusão de lesão total de manguito rotador, 31 com lesão parcial $(43 \%)$ e sete pacientes $(9,8 \%)$ tiveram laudo normal (Figura 3).

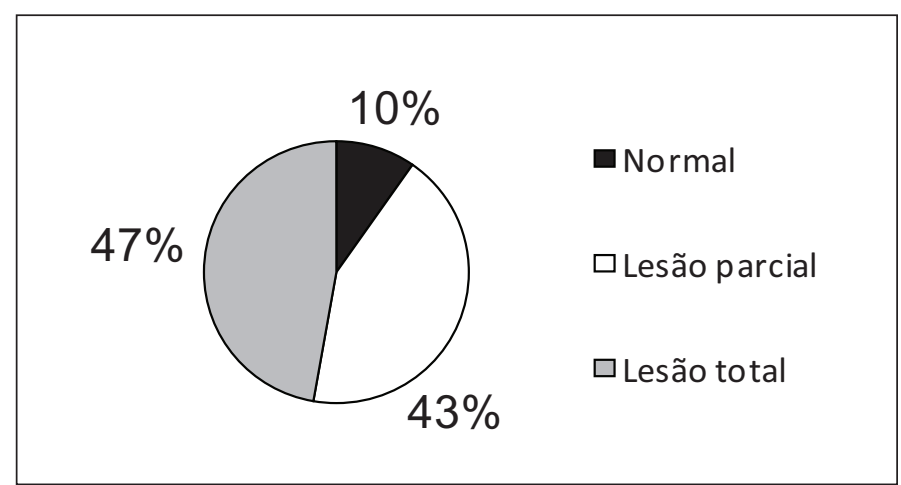

Figura 3 - Distribuição dos pacientes segundo a presença de alterações na ressonância nuclear magnética (valores percentuais aproximados).

Durante os 147 procedimentos cirúrgicos, encontramos 38 pacientes sem lesão de manguito rotador $(25,8 \%), 37(25,2 \%)$ com lesão parcial e 72 (49\%) com lesão total.

$\mathrm{Na}$ avaliação estatística da comparação entre a radiografia e o procedimento cirúrgico (padrão ouro), encontramos que, dos 131 pacientes que apresentavam alguma alteração na radiografia, 34 (26\%) tinham lesão parcial de manguito e 60 (45,8\%), lesão total. Assim, para comparar lesão parcial do manguito rotador com imagens de alterações na grande tuberosidade, a radiografia apresentou sensibilidade de $91,8 \%$ e especificidade de $11,8 \%$ apenas. Dos 13 pacientes com imagem osteofitária anteroinferior ("sinal do espelho"), todos tiveram alguma lesão tendínea, sendo três (24\%) com lesão parcial e 10 (76\%) com lesão total. Desta forma, temos que quando a radiografia apresenta "sinal do espelho", a sensibilidade e especificidade para lesão total do manguito 
rotador é de $13,8 \%$ e $96 \%$, respectivamente. Com base nestes dados, encontramos uma sensibilidade de 13,8\%, especificidade de $2,6 \%$ e acurácia de $30 \%$ quando comparamos o método radiográfico com qualquer tipo de lesão de manguito rotador (Tabela 1).

$\mathrm{Na}$ comparação entre a ultrassonografia e o procedimento cirúrgico, dos 43 pacientes que tiveram laudo ultrassonográfico de lesão parcial, 14 (32,5\%) tinham mesmo lesão parcial durante a cirurgia, mesmo número encontrado também com lesão total (14 pacientes). Assim, na análise dos pacientes com lesão parcial diagnosticados pela ultrassonografia e avaliados posteriormente durante a cirurgia, encontramos sensibilidade de $63,6 \%$ e especificidade de $63,2 \%$. Entretanto, nos 36 pacientes com laudo de lesão total visto à ultrassonografia, 30 pacientes $(83,5 \%)$ tinham lesão com ruptura total do manguito rotador evidenciada durante o procedimento cirúrgico, contra apenas quatro pacientes sem alterações e dois com lesão parcial. Quando analisamos a relação entre ruptura completa do manguito na ultrassonografia e comprovação no procedimento, obtivemos sensibilidade de $57,6 \%$ e especificidade de $87,7 \%$. Com base nestes resultados, este método apresentou uma sensibilidade de 57,6\%, especificidade de 29,6 e acurácia de $51,4 \%$ quando se comparou a totalidade dos dados para existência de qualquer tipo de lesão do manguito rotador (Tabela 2).
Analisando os resultados da comparação entre ressonância nuclear magnética e cirurgia, dos sete pacientes que tiveram conclusão do exame como "sem lesão", encontramos quatro pacientes (57\%) com manguito rotador íntegro durante a cirurgia, $2(28,5 \%)$ com lesão parcial e $1(14,5 \%)$ com lesão total. Assim, obtivemos uma sensibilidade de $22,2 \%$ e especificidade de $94,3 \%$ quando o laudo da ressonância era "normal" para lesão de manguito. Com relação aos 30 pacientes que apresentavam laudo da ressonância com lesão parcial de manguito, encontramos $12(38,7 \%)$ sem lesão vista no transoperatório, 15 (48,3\%) com lesão parcial confirmada e três (13\%) com lesão total. Com base nestes dados, encontramos uma sensibilidade de $65,2 \%$ e especificidade de $68,7 \%$ para ressonância com lesão parcial do manguito rotador. Ao analisarmos os 34 pacientes com lesão total apresentada na ressonância magnética, encontramos dois pacientes $(6 \%)$ com tendões sem alterações, seis $(17,6 \%)$ com lesão parcial confirmada e 26 (76,4\%) com lesão total. Portanto, os resultados obtidos demonstram sensibilidade de $86,6 \%$ e especificidade de $80,4 \%$ quando o diagnóstico da ressonância é de ruptura completa do manguito. Desta forma, a análise destes dados revela uma sensibilidade de $86,6 \%$, especificidade de $22,2 \%$ e acurácia de $63,3 \%$ deste método comparado com o padrão ouro quando se trata de qualquer uma das lesões do manguito rotador, ou quando o resultado da mesma é considerado normal para essa afecção (Tabela 3 ).

Tabela 1 - Avaliação dos achados sugestivos de lesão do manguito rotador vistos à radiografia nos pacientes submetidos ao tratamento cirúrgico (padrão ouro) em valores absolutos.

\begin{tabular}{|c|c|c|c|c|c|c|c|}
\hline \multicolumn{3}{|c|}{ Procedimento cirúrgico } & \multicolumn{5}{|c|}{$\mathbf{R X}$} \\
\hline & & & Normal & Alterações Gran & Tubérculo & $\begin{array}{l}\text { Imagem em } \\
\text { espelho }\end{array}$ & Total \\
\hline & \multicolumn{2}{|c|}{ Sem alteração } & 1 & \multicolumn{2}{|l|}{37} & 0 & 38 \\
\hline & \multicolumn{2}{|c|}{ Alteração parcial } & 0 & \multicolumn{2}{|l|}{34} & 3 & 37 \\
\hline & \multicolumn{2}{|c|}{ Alteração total } & 2 & \multicolumn{2}{|l|}{60} & 10 & 72 \\
\hline & \multicolumn{2}{|c|}{ Total } & 3 & \multicolumn{2}{|c|}{131} & 13 & 147 \\
\hline \multicolumn{2}{|c|}{ Resultados para qualquer lesão } & \multicolumn{2}{|c|}{$\begin{array}{c}\mathrm{X} \\
\text { Sem lesão de manguito }\end{array}$} & \multicolumn{2}{|c|}{$\begin{array}{l}\text { Alterações grande tuberosidade } \\
\qquad \mathrm{X}\end{array}$} & $\begin{array}{r}\text { Imagem e } \\
\text { Lesão total }\end{array}$ & $\begin{array}{l}\text { elho } \\
\text { nguito }\end{array}$ \\
\hline Acurácia & 0,306 & Acurácia & 0,734 & Acurácia & 0,319 & Acurácia & 0,557 \\
\hline Sensibilidade & 0,138 & Sensibilidade & 0,981 & Sensibilidade & 0,918 & Sensibilidade & 0,138 \\
\hline Especificidade & 0,026 & Especificidade & 0,026 & Especificidade & 0,118 & Especificidade & 0,96 \\
\hline Taxa de falso + & 0,230 & Taxa de falso + & 0,666 & Taxa de falso + & 0,740 & Taxa de falso + & 0,230 \\
\hline Taxa de falso - & 0,666 & Taxa de falso - & 0,345 & Taxa de falso - & 0,187 & Taxa de falso - & 0,462 \\
\hline Valor preditivo + & 0,769 & Valor preditivo + & 0,333 & Valor preditivo + & 0,259 & Valor preditivo + & 0,769 \\
\hline Valor preditivo - & 0,537 & Valor preditivo - & 0,743 & Valor preditivo - & 0,812 & Valor preditivo - & 0,537 \\
\hline
\end{tabular}


Tabela 2 - Avaliação dos achados sugestivos de lesão do manguito rotador vistos à ultrassonografia nos pacientes submetidos ao tratamento cirúrgico (padrão ouro) em valores absolutos.

Procedimento cirúrgico

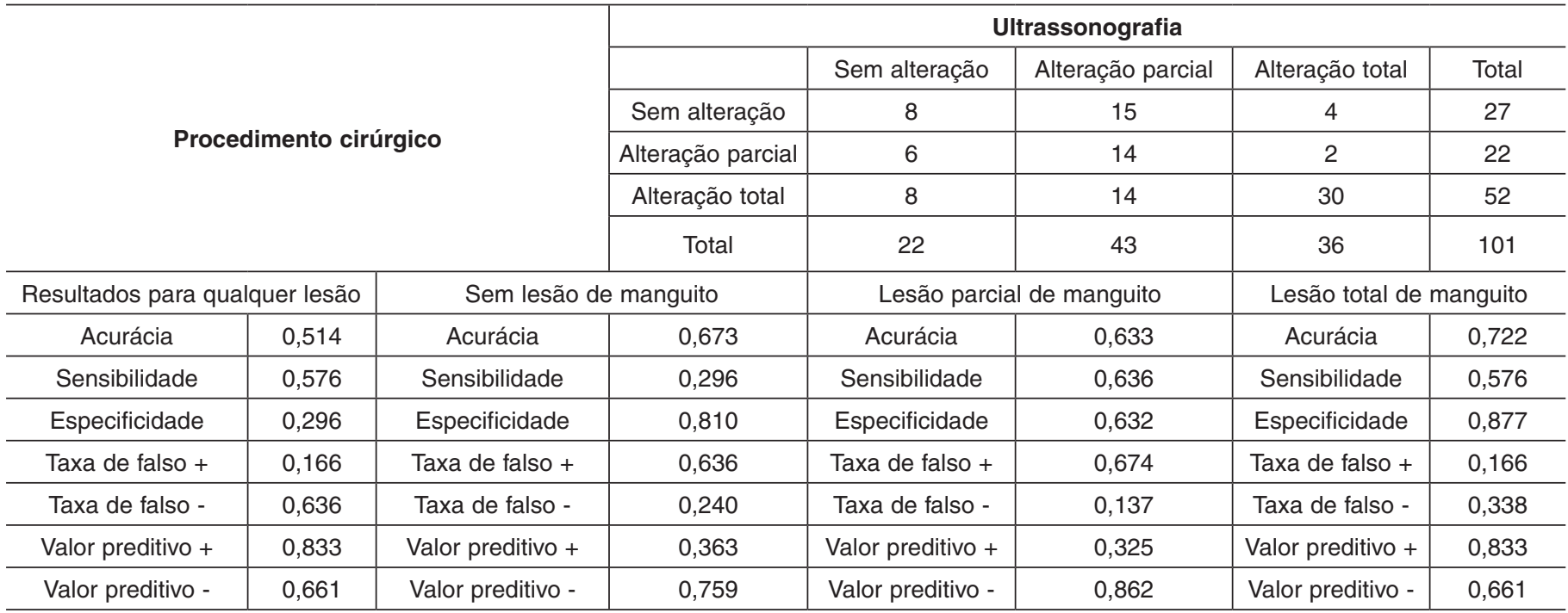

Fonte: Dados coletados de 2002-2007.

Tabela 3 - Avaliação dos achados sugestivos de lesão do manguito rotador vistos à ressonância nuclear magnética nos pacientes submetidos ao tratamento cirúrgico (padrão ouro) em valores absolutos.



\section{Ressonância nuclear magnética}

Fonte: Dados coletados de 2002-2007.

A visualização dos resultados das análises estatísticas pode ser feita mediante a observação do gráfico no qual se pode comparar a sensibilidade, especificidade, acurácia e os demais índices nos três métodos diagnósticos (Figura 4).

\section{DISCUSSÃO}

Aproximadamente $60 \%$ de todas as doenças do ombro são atribuídas às lesões do manguito rotador. Os estudos artroscópicos sugerem que 10\% dos casos de ombro doloroso sejam por lesão de espessura total, enquanto os $90 \%$ restantes sejam por lesões parciais ${ }^{(13)}$.

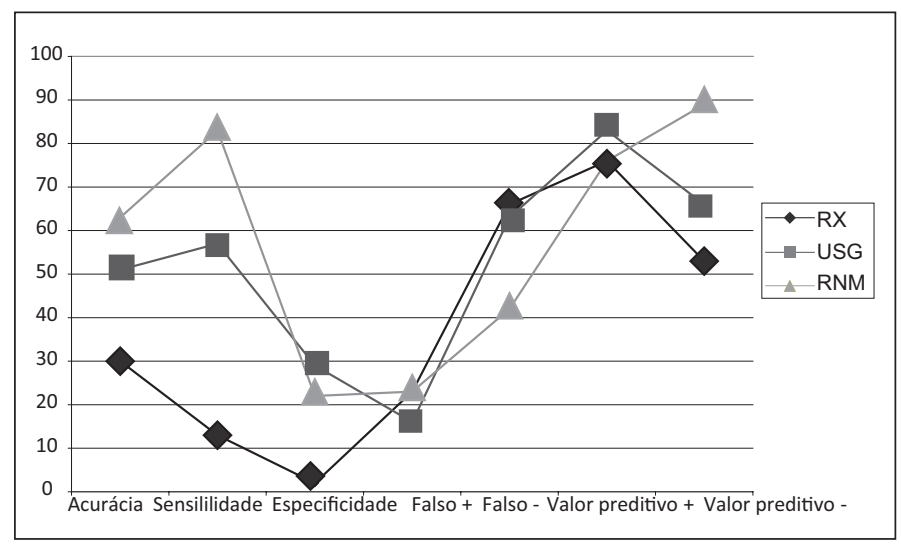

Figura 4 - Marcadores estatísticos dos três métodos diagnósticos avaliados no trabalho. 
Atualmente, tanto a ultrassonografia quanto a ressonância nuclear magnética são métodos usados no diagnóstico das lesões do manguito rotador, e não existe um método eleito claramente como de escolha ${ }^{(3,14)}$. Entretanto, a melhora nos transdutores e o preço mais acessível da ultrassonografia têm feito deste um bom método inicial de pesquisa frente a um paciente com dor no ombro e suspeita da doença $a^{(4,15)}$.

A revisão da literatura mostra uma variação grande dos resultados da ultrassonografia na identificação de rupturas dos tendões do manguito rotador, com relatos de sensibilidade superior a $90 \%$ em alguns trabalhos, assim como apresentam resultados muito menos expressivos em outros artigos ${ }^{(16-20)}$. Isso ocorre por alterações nos aparelhos de ultrassom, nas diferentes populações estudadas e na experiência dos ultrassonografistas. Em nosso trabalho encontramos uma sensibilidade de 57,6\% e especificidade de $29,6 \%$ no geral. No entanto, ao tratar especificamente lesões totais, o método apresentou uma sensibilidade de $57,6 \%$ e especificidade de $87,7 \%$; e, ao tratar lesões parciais, encontramos $63,6 \%$ sensibilidade e $63,2 \%$ de especificidade. Sabemos da maior dificuldade no diagnóstico das lesões parciais e das limitações de sensibilidade do método ${ }^{(21)}$, mas acreditamos que os resultados estão de acordo com a literatura estudada.

$\mathrm{O}$ risco de se utilizar um método com baixos índices de sensibilidade, como encontrado na utilização do ultrassom, é a não indicação de tratamento cirúrgico pelo fato de se tratarem de falsos-negativos ${ }^{(22)}$.

Em lesões maciças do manguito rotador, os tendões estão retraídos abaixo do acrômio e existe contato direto da cabeça umeral com o deltoide. No entanto, quando a bursa subacromial e região peribursal estão muito espessadas, a ultrassonografia pode interpretar esse dado como tendão íntegro e aí teremos um falsonegativo $^{(13,23,24)}$. Nosso valor preditivo positivo foi de $83,3 \%$ e valor preditivo negativo foi de $66,1 \%$, similares aos dados de outros autores ${ }^{(24,25)}$.

A ressonância nuclear magnética é um exame limitado quando tratamos de pacientes obesos, quando existem artefatos causados pela mobilidade e pela não tolerância por pacientes claustrofóbicos, além de ter certas restrições quando existe algum implante metálico. É relatada também uma acurácia menor no diagnóstico de lesões de espessura parcial em comparação às lesões de espessura total ${ }^{(26)}$. Em nosso estudo, encontramos taxas de $86,6 \%$ e $80,4 \%$ de sensibilidade e especificidade, respectivamente, quando tratamos apenas de pacientes com ruptura completa do manguito rotador, tendo como acurácia um resultado de $83,09 \%$. O grupo de pacientes no qual o diagnóstico foi de lesão parcial, encontramos $65,2 \%$ de sensibilidade e $68,7 \%$ de especificidade, com acurácia de $67,5 \%$. A literatura apresenta resultados que variam de $82 \%-100 \%$ de sensibilidade e de $79-100 \%$ de especificidade para lesões tendíneas completas ${ }^{(7,16,19)}$.

Martín-Hervás et al ${ }^{(22)}$ encontraram alta sensibilidade (100\%) no diagnóstico de lesões parciais utilizando a ressonância magnética, porém com baixa taxa de especificidade $(29,8 \%)$, o que resultou em poucos falsosnegativos; porém, quando a lesão era presente no exame, não havia certeza de sua presença, com altas taxas de falsos-positivos, obtendo um valor preditivo positivo de 19,5\%. Em nosso estudo, encontramos taxas mais elevadas de especificidade para lesões parciais, encontrando um valor preditivo positivo de $50 \%$. O valor preditivo positivo na realização de ressonância magnética para qualquer paciente deste estudo, ou seja, levando-se em consideração a presença ou ausência de qualquer lesão, foi de $76,4 \%$.

A ressonância magnética se mostra superior aos demais exames quando estamos avaliando a qualidade dos tendões do manguito, pois evidencia o nível de retração e a atrofia dos mesmos.

Quando estudamos pacientes com diagnóstico de rupturas completas do manguito rotador obtivemos resultados parecidos, com $86,6 \%$ sensibilidade da ressonância magnética contra $57,6 \%$ da ultrassonografia, enquanto na avaliação da especificidade encontramos $80,4 \%$ e $87,7 \%$, respectivamente. Assim sendo, podemos supor que dentre os pacientes estudados, quando se trata de lesões completas do manguito, a ressonância apresentou maior capacidade de diagnosticar os doentes, enquanto que a ultrassonografia foi melhor para excluir os que não apresentavam a lesão completa. Comparando a acurácia dos dois métodos quando analisamos ruptura completa dos tendões do manguito, a ressonância apresentou o valor de 83,09\% contra $67,3 \%$ da ultrassonografia. Esses dados são semelhantes aos encontrados nos relatos da literatura ${ }^{(22)}$.

Analisando as rupturas parciais de nossa casuística e seguindo a comparação dos métodos, encontramos sensibilidade de $63,6 \%$ da ultrassonografia contra $65,2 \%$ da ressonância. Ao compararmos a especificidade, chegamos aos valores de 63,2\% e 68,7\%, respectivamente. Esses dados mostram uma diferença menor entre os dois exames complementares a favor da ressonância em comparação à análise das rupturas totais apresentada acima. 
$\mathrm{Na}$ avaliação da acurácia dos métodos estudados, encontramos que tanto a ultrassonografia quanto a ressonância magnética apresentaram elevada acurácia, com resultados pouco mais expressivos para este exame do que para aquele. A radiografia é um método que apresentou resultados menos favoráveis e sua utilização se torna importante quando encontramos o sinal do espelho, que apresentou $96 \%$ de especificidade para ruptura completa. Este dado indica que quando encontramos a osteofitose na região anteroinferior do acrômio, existe alta probabilidade de o paciente apresentar lesão de espessura completa do manguito rotador. Assim sendo, a radiografia se apresenta como um exame importante na investigação do ombro doloroso com suspeita de lesão de manguito.

\section{CONCLUSÃO}

A ressonância nuclear magnética e a ultrassonografia se mostraram métodos confiáveis apresentando elevada acurácia no diagnóstico das lesões do manguito rotador.

A radiografia se mostrou um exame específico quando apresentou sinal do espelho, sendo ferramenta de fácil obtenção e permanecendo consagrada no diagnóstico das lesões do manguito.

\section{REFERÊNCIAS}

1. Andrade RP, Corra Filho MR, Queiroz BC. Lesões do manguito rotador. Rev Bras Ortop. 2004;39:621-36.

2. Volpon JB, Erdmann K, Nisiyama CY, Monteiro CR. Ruptura do manguito rotador do ombro em pacientes com pinçamento crônico. Rev Bras Ortop. 1989;24(4):111-4.

3. Bachmann GF, Melzer C, Heinrichs CM, Möhring B, Rominger MB. Diagnosis of rotator cuff lesions: comparison of US and MRI on 38 joint specimens. Eur Radiol. 1997;7(2):192-7.

4. Read JW, Perko M. Shoulder ultrasound: diagnostic accuracy for impingement syndrome, rotator cuff tear, and biceps tendon pathology. J Shoulder Elbow Surg. 1998;7(3):264-71.

5. Paavolainen $P$, Ahovuo J. Ultrasonography and arthrography in the diagnosis of tears of the rotator cuff. J Bone Joint Surg Am. 1994;76(3):335-40.

6. Teefey SA, Middleton WD, Yamaguchi K. Shoulder sonography. State of the art. Radiol Clin North Am. 1999;37(4):767-85.

7. Iannotti JP, Zlatkin MB, Esterhai JL, Kressel HY, Dalinka MK, Spindler KP. Magnetic resonance imaging of the shoulder. Sensitivity, specificity, and predictive value. J Bone Joint Surg Am. 1991;73(1):17-29.

8. Uri DS. MR imaging of shoulder impingement and rotator cuff disease. Radiol Clin North Am. 1997;35(1):77-96.

9. Yamaguchi K. Mini-open rotator cuff repair: an updated perspective. Instr Course Lect. 2001;50:53-61.

10. Yamaguchi K, Levine WN, Marra G, Galatz LM, Klepps S, Flatow EL. Transitioning to arthroscopic rotator cuff repair: the pros and cons. Instr Course Lect. 2003;52:81-92.

11. Warner JJ, Goitz RJ, Irrgang JJ, Groff YJ. Arthroscopic-assisted rotator cuff repair: patient selection and treatment outcome. J Shoulder Elbow Surg 1997;6(5):463-72.

12. Zlatkin MB, lannotti JP, Roberts MC, Esterhai JL, Dalinka MK, Kressel HY, et al. Rotator cuff tears: diagnostic performance of MR imaging. Radiology. 1989;172(1):223-9.

13. Van Holsbeeck MT, Craig JG. Bouffard JA, Shirazi KK. Shoulder pain. Radiol Soc North Am. 1996;117-23.

14. Torstensen ET, Hollinshead RM. Comparison of magnetic resonance imaging and arthroscopy in the evaluation of shoulder pathology. J Shoulder
Elbow Surg. 1999;8(1):42-5.

15. Erickson SJ. High-resolution imaging of the musculoskeletal system. Radiology. 1997;205(3):593-618.

16. Burk DL Jr, Karasick D, Kurtz AB, Mitchell DG, Rifkin MD, Miller CL, et al. Rotator cuff tears: prospective comparison of MR imaging with arthrography, sonography, and surgery. AJR Am J Roentgenol. 1989;153(1):87-92.

17. Crass JR, Craig EV, Thompson RC, Feinberg SB. Ultrasonography of the rotator cuff: surgical correlation. J Clin Ultrasound. 1984;12(8):487-91.

18. Mack LA, Matsen FA 3rd, Kilcoyne RF, Davies PK, Sickler ME. US evaluation of the rotator cuff. Radiology. 1985;157(1):205-9.

19. Nelson MC, Leather GP, Nirschl RP, Pettrone FA, Freedman MT. Evaluation of the painful shoulder. A prospective comparison of magnetic resonance imaging, computerized tomographic arthrography, ultrasonography, and operative findings. J Bone Joint Surg Am. 1991;73(5):707-16.

20. Middleton WD, Reinus WR, Totty WG, Melson CL, Murphy WA. Ultrasonographic evaluation of the rotator cuff and biceps tendon. J Bone Joint Surg Am. 1986;68(3):440-50.

21. Brenneke SL, Morgan CJ. Evaluation of ultrasonography as a diagnostic technique in the assessment of rotator cuff tendon tears. Am J Sports Med. 1992;20(3):287-9.

22. Martín-Hervás C, Romero J, Navas-Acién A, Reboiras JJ, Munuera L. UItrasonographic and magnetic resonance images of rotator cuff lesions compared with arthroscopy or open surgery findings. J Shoulder Elbow Surg. 2001;10(5):410-5.

23. Middleton WD. Ultrasonography of the shoulder. Radiol Clin North Am. 1992;30(5):927-40.

24. Thain LF, Adler RS. Shoulder: rotator cuff and long head of biceps tendons. In: Chhem RK, Cardinal E, editors. Guidelines and gamuts in musculoskeletal ultrasound. New York: Wiley-Liss; 1999. p. 39-72.

25. Swen WA, Jacobs JW, Algra PR, Manoliu RA, Rijkmans J, Willems WJ, et al. Sonography and magnetic resonance imaging equivalent for the assessment of full-thickness rotator cuff tears. Arthritis Rheum. 1999;42(10):2231-8.

26. Thomazeau H, Boukobza E, Morcet N, Chaperon J, Langlais F. Prediction of rotator cuff repair results by magnetic resonance imaging. Clin Orthop Relat Res. 1997;(344):275-83. 


\section{ANEXO 1. Protocolo do serviço de cirurgia do ombro e cotovelo.}

Nome: $\mathrm{M} \square \mathrm{F} \square$ Idade: Lado: $\mathrm{D} \square \mathrm{E} \square$

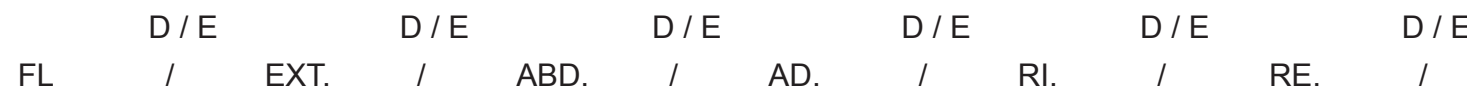

Convênio e $\mathrm{n}^{\mathrm{o}}$ : Profissão:

Instabilidade: Carga/deslizamento $\square \quad$ Apreensão $\square \quad$ Relocação $\square \quad$ Deslocamento espontâneo $\square$ Sulco $\square \quad$ Fukuda $\square \quad$ Outras articulações

Porção Longa Bíceps: Palpável $\square \quad$ Yergason $\square \quad$ Speed $\square \quad$ Ludington $\square \quad$ O’Brien $\square$ Supraespinhoso: Sinal impacto $\square \quad$ Teste impacto $\square \quad$ Arco doloroso $\square \quad$ Yokum $\square \quad$ Jobe $\square$ Infra e RM: Hawkins $\square \quad$ Teste infra $\square \quad$ Patte para infra $\square \quad$ Cancela $\square \quad$ Drop arm test $\square$ Subescapular: Gerber $\square \quad$ Abdominal press $\square$

Acromioclavicular: Palpação dolorosa $\square \quad$ Cross arm Coracóide: Hawkins Kennedy $\square$

Neurológico: C5 $\square \quad$ C6 $\square \quad$ Axilar $\square \quad$ Musculatura $\square \quad$ Supraespinhoso $\square$ Cervical : Artrose $\square \quad$ Radiculopatia $\square \quad$ Desf. torácico $\square \quad$ Outros

Restante: Mediano Ulnar Radial Outros
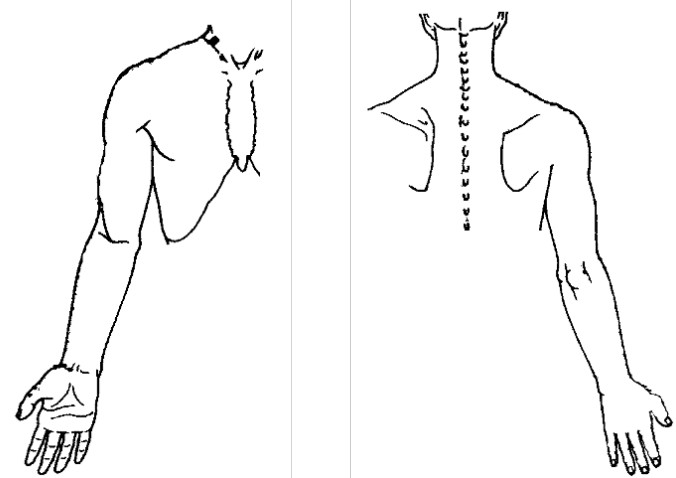

Exames: Radiografia

Ultrassonografia

Ressonância

Cirurgia
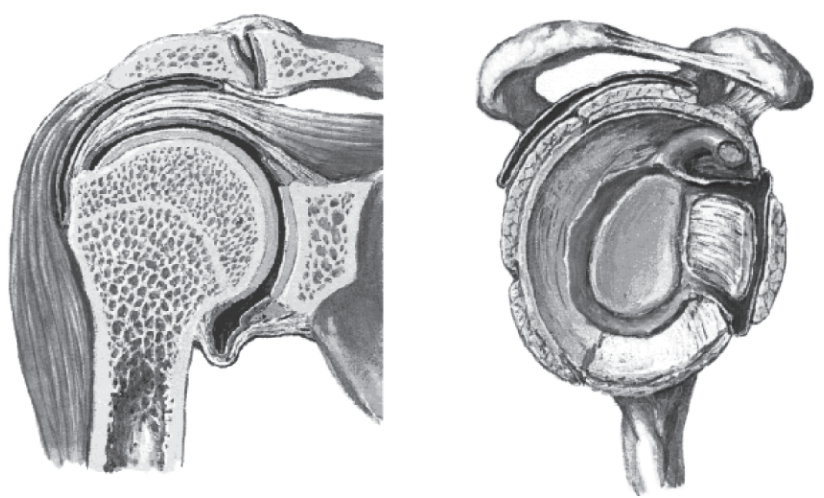